\title{
A MOVABLE CHARGING UNIT FOR GREEN MOBILITY
}

\author{
Eiman Y. ElBanhawy ${ }^{\mathrm{a}} *$, Khaled Nassar ${ }^{\mathrm{b}}$ \\ ${ }^{a}$ Engineering and Environment Faculty, Northumbria University, Newcastle Upon Tyne, UK- \\ eiman.elbanhawy@northumbria.ac.uk \\ ${ }^{\mathrm{b}}$ Construction and Architecture Engineering Department, American University in Cairo, Egypt - \\ Knassar@aucegypt.edu
}

KEY WORDS: Batteries swapping, electric vehicles, electric vehicles range anxiety, charging behaviour, shortest path algorithm problem, movable charging unit,

\begin{abstract}
:
Battery swapping of electric vehicles (EVs) matter appears to be the swiftest and most convenient to users. The existence of swapping stations increases the feasibility of distributed energy storage via the electric grid. However, it is a cost-prohibitive way of charging. Early adaptors' preferences of /perceptions about EV system in general, has its inflectional effects on potential users hence the market penetration level. Yet, the charging matter of electric batteries worries the users and puts more pressure on them with the more rigorous planning-ahead they have to make prior to any trip. This paper presents a distinctive way of charging. It aims at making the overall charging process at ease. From a closer look into the literature, most of EVs' populations depend on domestic charge. Domestic charging gives them more confidence and increases the usability factor of the EV system. Nevertheless, they still need to count on the publically available charging points to reach their destination(s). And when it comes to multifamily residences, it becomes a thorny problem as these apartments do not have a room for charging outlets. Having said the irritating charging time needed to fatten the batteries over the day and the minimal average mileage drove daily, hypothetically, home delivery charging (Movable Charging Unit-MCU) would be a stupendous solution. The paper discusses the integration of shortest path algorithm problem with the information about EV users within a metropolitan area, developing an optimal route for a charging unit. This MCU delivers charging till homes whether by swapping batteries or by fast charging facility. Information about users is to be provided by the service provider of the neighbourhood, which includes charging patterns (timing, power capacity). This problem lies under the shortest path algorithms problem. It provides optimal route of charging that in return shall add more reliability and usability values and alleviate the charging/ limited range / daily planning anxieties. The model is in a very preliminary stage of development, future work is needed to elaborate on the model and developing a complete feasibility study.
\end{abstract}

\section{INTRODUCTION}

\subsection{Introduction}

This paper aims at discussing alternative way of charging electric vehicles (EVs). It presents a more useful and helpful idea of charging pattern in order to ease the charging process. The rationale behind the proposed charging technique came from the users' feedback and market penetration level. Market participants need to fully understand consumers' perceptions and preferences in order to make the right investments at the right time (McGuinness, 2011). In the presence of the concurrent environmental concerns regarding the $\mathrm{Co} 2$ and greenhouse gases (GHG) emissions, many developed and industrial countries have been taking initiatives to promote low carbon emissions vehicles. These active regions believe that finding alternative green means of transportation would positively have its resilient effect on the overall carbon footprint. In these active regions yet the EV market is hindered with several factors. One of these hurdles revolves around a simple daily question raised by the users, where to charge my EV? This question takes place due to the electric vehicles range anxiety, EVRA. The EVRA is a very common syndrome that happens to EVs' drivers. The authors have been working on the area of simulating EVs systems examining the integration of charging points within metropolitan areas (ElBanhawy et al,
2012A). The simulation aims at depicting the whole picture of the system and trying to develop a planning assistant tool that can assist policy makers and planners in planning for new charging points (CPs) to support the future users (ElBanhawy et al, 2012B); (ElBanhawy et al, 2013). Vehicular simulation solution has been the selected way to depict the phenomenon of users' charging patterns.

\subsection{Market Penetration Level}

In Europe and with a particular strategic focus on private and non-commercial electric cars, the total number of registered cars can indicate the level of market penetration. In the first half of 2011, the total EVs registrations were 5,222 (JATO, 2011) which depicts the EV niche market that is in a real need of expansion and creation of conditions for growth for the mass adoption (Graham-Rowe, 2012). The market is hampered by many factors e.g. cost, range, capacity, visual appeal (GrahamRowe, 2012) speed, and lack of recharging infrastructure integration (Garling, 2001); (Hatton et al., 2009). The higher upfront price of EV compared to conventional car is one of the considerable barriers by potential users (Garling, 2001); nevertheless, the degree of urban geography, market maturity and infrastructure have their effects on market growth (JATO, 2011) as well.

\footnotetext{
* Corresponding author. This is useful to know for communication with the appropriate person in cases with more than one author.
} 
Several factors pertinent to EVs' battery range appear to influence users' anxiety during driving; known as EVRA (Nilsson, 2011). It is believed that this anxiety hinders the EV market expansion (DfT, 2011). EVRA basically exists due to the short full-electric range the EVs have (HMGovernment, 2011). Full-electric range is the maximum distance a vehicle could travel without a need of charge (Eppstein, 2011). Therefore, it can be said that providing accessible and high visible charging network (hard and soft infrastructure (Beeton, 2011) generates interest amongst consumers and encourages uptake (Element Energy Ltd, 2009). Towards developing a unified ecosystems and smart cities, investigating and predicting the consumers' response is a significant challenge EV marketers are facing (Beeton, 2011); (Strahan, 2012). EV market is on the verge of expansion and maturity.

\subsubsection{Optimization-based and heuristic-based techniques}

This paper uses optimization based computing solution. This solution has been used widely in construction site layout facilities problems. This application was due to the importance of having appropriate site layout of temporary facilities to enhance productivity and safety on construction sites. It is a complex problem and in recent years researchers have attempted to solve using a variety of optimization-based and heuristic-based techniques. In a study conducted by Hegazy, a genetic-algorithm based model for site layout planning was presented and analyzed. This model which is called (EVOSITE) because of the use of evolutionary algorithms, has several advantages. It is applied to any user-defined site shape; it accounts for the user preference in the relative closeness among the facilities; and it incorporates a genetic algorithm procedure to search for the optimum layout in a manner that simulates natural evolution. EVOSITE uses excel Microsoft office intuitive spreadsheet. The representation of site and the facilities are in a two dimensional grid. GA is applied to automate the evolution of the layout solutions. (Tarek Hegazy, 1999); (ElBanhawy, 2011).

\subsection{Train of thoughts}

The paper addresses the following points:

1. Discussing and finding the winning strategies for charging;

2. Presenting a hypothetical approach to deliver charging service;

3. Examining the factors that would affect charging delivery.

The paper is structured to start with the social logic of charging showing the focus of the study and giving an overview of the EVs market. This is followed by the mechanism of the proposed approach which is finding the shortest/ optimal path problem solving technique. The hypothetical model is presented showing the parameters, variables, and constraints ending with the discussion and conclusion.

\section{SOCIAL LOGIC OF CHARGING}

\subsection{Focus of the study}

This study strategically focuses on both private electric cars. The total number of registered electric cars can indicate the market penetration. In Europe, in the first half of 2011, the total EVs registrations were 5,222 (JATO, 2011). This implies a niche market that is in a real need of expansion and creation of conditions for growth for the mass adoption of EVs when 'early adopters' may not represent 'mainstream consumers' (E. Graham-Rowe, 2012); (J. Gould and T. Golob, 2012). Statistics showed that most of the users rely on domestic charging as this keep them more secured (McDonald, 2012). This study likewise other sides focusing on those who do not rely on domestic charging, studies all possible means of charging pattern that will better work for the users. The system usability is one of the crucial

\subsection{Charging Services Market}

One of the primary obstacles to expanding the EV market in large urban centres around the world is the charging process. The charging points are marked at three levels of power delivery for the current commercially available technology. Level 1 which is $(110 / 120 \mathrm{~V})$ at $15-20$ Amps and level 2 (220/240V) at 40 Amps that charges in less time, or the DC fast charging $(480 \mathrm{~V})$ option that can fill up the battery in a fraction of an hour (inductive or deductive). The three options are there for charging though the first two are the best suited for residential applications. Charging service might take place at homes/ multifamily residence (domestic), on street (publically available charging), off street (at work, parking lot, private zones).The fast-charging option suites more with the in-street and off-street parking. Yet, it is considered as a cost prohibitive option for residential applications and it sometimes goes beyond the charging needs of most city drivers.

Charging frequency alternates as it is a function of distance driven, electricity prices, diving style and pattern, load and external conditions. Drivers who are depleting their batteries on a daily basis need to charge daily and probably domestically while those who drive less comparatively can charge on street or at work and not even on a daily basis (Peterson, 2011).

\subsection{Swapping batteries}

Another option of charging the vehicles is to swap the flat battery with a fully charged one which is an expensive option though more convenient, the battery swapping. This charging pattern is currently being championed by several service providers in different countries, Israel is notably considered as a leader in this technique. In 2011, swapping EVs' batteries has showed up to be an alternative way of recharging. In Israel, the drivers have to direct themselves to the nearest available charging point, and all it takes is an RFID card to enter the station, and robots from below swap out the empty battery for a fully charged alternative while the car passenger would occupy themselves for three minutes (Schluman, 2011).

\subsection{Charging pattern trend}

A global study by Accenture (NYSE: ACN), a study of over 7000 people in 13 countries, found that 62 percent would reject battery swapping, where empty batteries are quickly replaced at service stations for fully charged ones, preferring to plug in their car to recharge the battery. This limits the opportunity for charging off peak, when battery swapping companies would most likely refuel batteries. It also revealed that consumers need more supportive charging infrastructure in order to adopt fully EVs. Only 29 percent of car drivers would buy EV where the rest prefer plug-in hybrid EVs (PHEVs), which run on gasoline / diesel once the battery runs low. 85 percent say fully electric PEVs have insufficient battery range to cover their daily driving needs. But 83 percent cite the insufficient availability of 
charging points and 70 percent think charging times for full plug-in EVs are too long (McGuinness, 2011).

\subsection{Dilemma of on-street parking}

Domestic charging has been always the common choice of early adaptors as it ensures having a full battery to start the journey. Most EV drivers charge nightly or bi-nightly basis. This is also due to the very limited available time for charging through the day time, finding the nearest and available charging point with the commercial and technical compatibility measures. The study showed that 55 percent would only plug in their PEV when they need to charge up, rather than whenever they park. This behavior could result in less predictable charging patterns and could reduce the demand for public charging infrastructure (McGuinness, 2011).The problem does exist when it is a multifamily residences (i.e. apartments and condominiums) where insufficient number of parking spaces, constrained electrical room capacity, expensive installation costs and multiple EV users make it more thorny and does not tip the balance in favour of EV market penetration (Peterson, 2011). The problem is even more stubborn when it comes to existing neighbourhood and housing stock. These buildings are not designed to accommodate EVs.

\section{ALTERNATIVE MEAN OF CHARHING-SHORTEST PATH ALGORITHM PROBLEM}

To see the EV a viable long term transportation alternative, stakeholders and market participants have to circumvent the thorny matter of charging service. Shedding lights on the above, this paper presents an alternative way of charging that satisfies the following conditions: (1) less time to replenish the battery via providing level 1 and 2, (2) convenient time to charge as being available during the day, (3) convenient location as being close to their homes, (4) provides both swapping and charging options. The proposed solution aims at delivering charging service at the users' footsteps. Having said that most of the EVs' drivers count on the domestic charging, yet if they find an easier/ cheaper/ more convenient way to crank their motors, they will definitely go for. The whole notion is to design a charging hotspot. This hotspot is movable and versatile charging unit (MCU) as it serves the community wherever there is a not sufficient charging service or there is not an access to service. MCU will have a defined computed path and route. The route is calculated based on the shortest path algorithm where the algorithm is looking for the optimal path that the MCU should take. The best itinerary is chosen to cover the confined urban territory by:

1. Providing charging services to minorities not using domestic charging;

2. Serving as a back-up for those who charge domestically;

3. Allowing versatile charging options for all users (fast charging/ battery swapping);

Hence the users who are not counting on domestic charging, will no more need to look prior to their trips, to the nearest charging point in their vicinities(on street/off street), the publically available ones.

\subsection{Shortest path algorithm problem}

Engineering algorithms has attracted increasing attention during the last years (Wilhelm, 2005). Computing shortest paths is a base operation for many problems in traffic applications. The most prominent are certainly route planning systems for cars, bikers and hikers (Jabbar et al, 2005), or scheduled vehicles like trains and buses. These models are stem from real applications. Using realistic data is quite important as the performance of the algorithms strongly depends on the characteristics of the data. In shortest path problems, the algorithm works on reducing the search space by applying Dijkstra's algorithm. It tries to find the shortest path between nodes (intersections) and edges (links, segments, streets, avenues) starting from the source reaching the target (Wilhelm, 2005). In particular, this problem tries to solve many queries for the shortest paths; however, the present study is considered as a sub-problem of this case. It investigates only a one shortest path within the network; which is the route of the unit.

Within the model, edge lengths are not Euclidean distances. They are determined from travel times, thus representing our distance estimates. Reasonable (relative) location estimates are then obtained by embedding this graph in the plane such that edge lengths are approximately preserved. Parts of these results have been published previously in (Brandes, 2004).

\subsection{Travel Planning Systems}

A public transport model/graph might represent a network of trains, buses, and other scheduled vehicles which are the travel planning systems. These systems are considered as a restricted shortest path problem as the vehicle has to path through specific edges/nodes. The more details we add to the model, the more complex it becomes, for instance; connections, vehicle types, transfer times, or traffic days into account.

\section{MOVABLE CHARGING UNIT (MCU) MODEL}

In this section we introduce a hypothetical example of MCU model serves a residential district. The model starts with the layout designing of the MCU, defining the urban district, outlining the constraints and parameters, and finally running the algorithm and discussing the outcomes.

\subsection{Layout design of MCU}

The MCU is basically a charging hub, it is therefore contains 2 types of charging, fast charging (840 Volts) and batteries swapping. A schematic design of the unit from inside shows the two sections, figure (1). The right section has 4 outlets for fast charging which means the unit can serve 4 cars a time. In 40 minutes it can replenish $4 \mathrm{EVs}$ batteries that range between small battery capacity $(16 \mathrm{kWh})$ likewise Chevrolet Volt and large battery (24KWh) likewise Nissan Leaf. The left side of the unit is for batteries swapping. It has a fair number of batteries which are ready to be used. Special robot mechanism is attached to the unit to automatically replace the flat battery in the vehicles with a fully charged one, from those stored in the unit.

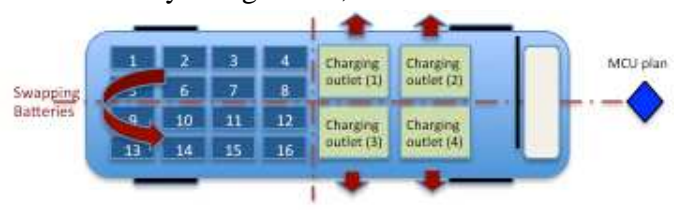

Figure1. MCUs Schematic Layout 


\subsection{The Experimental Neighbourhood District}

For the purpose of the study, a residential district of multifamily residences has been designed, figure (2). The district contains our zones need to have access to charging service. Each zone contains three blocks; each block has a number of EVs' owners, figure (3). The MCU has to serve each zone separately.
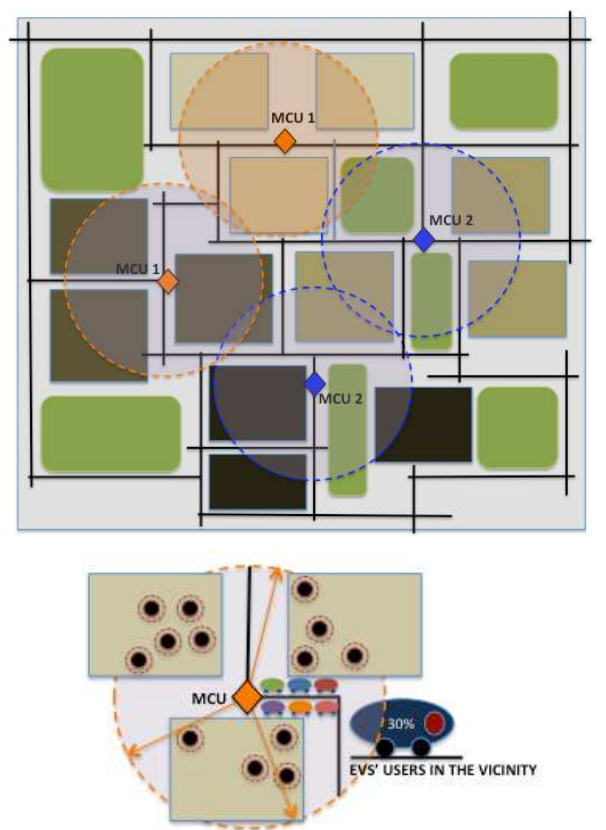

Figures $2 \mathrm{a}$ and 2b. Neighbourhood district layout/ MCU zone

Based on the selected path; the optimal possible locations for MCU to stop in each zone will be identified. The unit stops/locations (nodes) are picked to cover the EVs drivers who live in this zone. Based on former statistics and surveys, EVs' users are very minorities; however, we will consider that in each block there is a EV, figure (3). By this we ensure the MCU provides sufficient service in terms of geographical distance/ analysis.

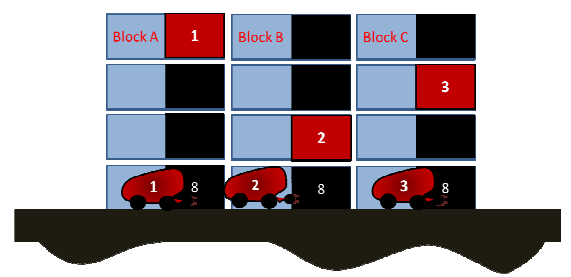

Figure3. MCUs path and location

\subsection{MCU Parameters and Constraints}

To design the MCU model, parameters and constraints have to be firstly clear. The first step is to convert the schematic layout of the district into a measurable graph in which we can identify the nodes and edges, source and target. Also, the travel cost tacked to each edge, which is the cost of travel from a node to another via this edge has to be identified and quantified. For a simple way of modelling, the layout is converted into an excel sheet, figure (4). Nodes are the intersection points. Roads are converted to segments, each segment starts and ends with a node. So in order to go from point $\mathrm{A}$ to $\mathrm{B}$, I have to pass by edge number 1 . The travel cost is identified on each edge, is the number of cells edge 1 is occupying.

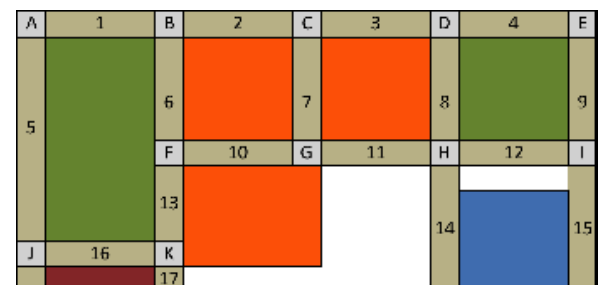

Figure4. District graph showing nodes and edges

The figure's colour scheme shows the four zones and the greenery within the district. The district has 29 nodes and 41 edges. The source is node $\mathrm{A}$ and the targets (shortest path container), nodes $\mathrm{Z}$ or node $\mathrm{Z} 3$.

The constraints can be summarized as follows:

1. MCU has to pass though all zones, figure $2 \mathrm{a}$;

2. The route can be taken by $2 \mathrm{MCU}$ in parallel so that the foru zones are served in 90 minutes in total.( check figure $2 \mathrm{a}$ for MCU1 and MCU2);

3. MCU stays a period of time which is equivalent to fully fast-charge a battery (45 minutes). The unit charges 4 cars in parallel (arrival and departure events);

4. The fourth slot/ electricity outlet is to be used by other EVs' drivers who live outside the zone;

5. The unit has to pass through the boundaries indicated in figure $2 \mathrm{~b}$. The more the stop is centred, the better service, the optimal the route.

\subsection{Hypothetical Journey within a neighbourhood}

All possible routes are calculated, which in total are 10 possible routes that meet the above constraints. The routes are tabled, table 1 , while the travel cost is calculated for each route.

Table 1. Possible routes

\begin{tabular}{|c|c|c|c|c|c|c|c|c|c|c|c|c|c|}
\hline Na & 51 & 52 & 53 & 54 & 55 & 56 & 57 & 58 & 59 & 510 & 511 & $\begin{array}{ll}512 & 513\end{array}$ & Metriciunits) \\
\hline 1 & $A B$ & $\mathrm{BF}$ & $\mathrm{FG}$ & $\mathrm{GH}$ & $\mathrm{HN}$ & NT & TS & $S R$ & RV & Ww & WZ1 & $\mathrm{Z1Z2} \quad \mathrm{Z2Z3}$ & 57 \\
\hline 2 & $A B$ & $\mathrm{BC}$ & CG & GH & $\mathrm{HN}$ & NM & MS & SR & RV & vw & $W \angle 1$ & $\angle 1 \angle 2 \angle 2 \angle 3$ & 57 \\
\hline 3 & $A B$ & BF & FK & KL & LM & M5 & ST & $T x$ & $x Z 2$ & Z2Z3 & & & 40 \\
\hline 4 & $A B$ & $B C$ & CG & GF & $\mathrm{FK}$ & $\mathrm{KL}$ & LM & MS & ST & $T X$ & $x Z 2$ & 2273 & 50 \\
\hline 5 & $A B$ & $\mathrm{BC}$ & CG & $\mathrm{GH}$ & $\mathrm{HN}$ & NT & $T x$ & $\mathrm{XT}$ & TS & $S R$ & RV & vu & 49 \\
\hline 6 & $A B$ & BF & FG & $\mathrm{GH}$ & $\mathrm{HN}$ & NM & MS & $S R$ & RV & vw & WZ1 & $\mathrm{Z1Z2}$ & 57 \\
\hline 7 & $A B$ & $B C$ & C. D & $\mathrm{DH}$ & $\mathrm{HN}$ & NT & TS & $S R$ & RV & WW & w71 & 717 & 51 \\
\hline 8 & $A B$ & $\mathrm{BF}$ & FK & $\mathrm{KL}$ & LM & $M N$ & NT & $T x$ & $x Z 2$ & $\mathrm{Z2Z3}$ & & & 40 \\
\hline 9 & $A B$ & BC & $C D$ & DH & $\mathrm{HN}$ & NM & M5 & SR & RV & WW & WZ1 & $\mathrm{Z1Z2} \mathrm{Z2Z3}$ & 57 \\
\hline 10 & $A B$ & $B C$ & CG & GF & FK & $\mathrm{KL}$ & LM & $\mathrm{MN}$ & NT & $T X$ & $X Y$ & $Y_{Z Z 2}$ & 50 \\
\hline
\end{tabular}

The table also shows very interesting outcomes, the coloured edges in each column indicates the most common edge that the 10 routes used to go through to reach the target. Results show that theoretically, there are two possible shortest routes to be taken as both of them have the same cost. However the optimal is the one that better serve the four zones as the unit can stop just in the middle of the zones' boundaries. 
Table 2a. Shortest routes Vs optimal route

\begin{tabular}{|c|cccccccccc|}
\hline Shartest MCU paths & & & & & & & Metric(units) \\
\hline 1 AB & BF & FK & KL & LM & MS & ST & TX & XZ2 & Z273 & 40 \\
\hline 2 AB & BF & FK & KL & LM & MN & NT & TX & XZ2 & Z273 & 40 \\
\hline \hline Optimal MCU path & & & & & & & & Metric(units) \\
\hline 1 AB & BF & FK & KL & LM & MN & NT & TX & XZ2 & Z273 & 40 \\
\hline
\end{tabular}

\section{DISCUSSION AND FUTURE WORK}

This paper aims at finding the winning strategies for charging green mobility. Due to the global concern of the $\mathrm{CO} 2$ and GHGs emissions, the world is shifting towards a smarter ecosystem where the roads emissions are kept to the least. EV industry is a promising solution to solve this thorny situation. Charging patterns have been one of the crucial matters that affect the market penetration and positioning among the other means of clean transportations. The paper presents an alternative way of charging EVs which makes the service at the users' footsteps. The model is based on finding the shortest/optimal path algorithm for a movable unit that provides charging services for users.

Yet, the proposed approach is cost-prohibitive due the batteriesswapping it provides. This is in addition to the service provider fee (administrative charge to use the UCM). However, this matter to be overcome with the roll out of intelligent infrastructure, creation of innovative service models and changes in consumer behaviour. All these positive transformations will shift the market towards the mainstream scale (Beeton, 2011). Future work is due by researchers to develop MCU business model in order to have a clear complete image of the feasibility study of MCU.

\section{REFERENCES}

A. Garling and J. Thøgersen, "Marketing of electric Vehicles", Busi. Strat. and the Envir., Vol. 10, pp. 53-65, 2001.

C.E. Hatton, S.K. Beela, J.C. Brezet, and Y.C. Wijnia, Charging stations for urban settings: the design of a product platform for electric vehicle infrastructure in Dutch cities, EVS24 International Battery, Hybrid and Fuel Cell Electric Vehicle Symposium, Stavanger, Norway, May 13-16, 2009.

David Strahan, "Dump the pump", NewScientist, Vol. 214, Issue 2865, pp. 34-38, May 2012.

D. Beeton, North East Electric Vehicle Roadmap. Report for the International Energy Agency's Implementing Agreement on Hybrid and Electric Vehicles. Newcastle upon Tyne: Urban Foresight Limited, 2011. Online access: http://e-mobilitynsr.eu/fileadmin/user_upload/downloads/info-

pool/NorthEast_EV_Roadmap.pdf

DfT, Transport energy and environment statistics. UK, 2011.

D. Peterson, Addressign Challendges to Electric Vehicle charging in multifamily residential buildings, Luskin Centre for Innocation, LCLA, June 2011

E. Graham-Rowe, B. Gardner, C. Abraham, S. Skippon, H. Dittmar, R. Hutchins, and J. Stannard, "Mainstream consumers driving plug-in battery-electric and plug-in hybrid electric cars: A qualitative analysis of responses and evaluations", Trans. Res. Part A: Pol. and Pract., Vol. 46(1), pp. 140-153, 2012.
ElBanhawy, E.Y., Visual Quality Integrated Cost Model for Developing Green Neighbourhoods: An Optimization Approach, Master's Thesis, American University in Cairo, 2011

ElBanhawy, E. Y., R. Dalton, E. M. Thompson, R. Kotter., 2012 A. A Heuristic Apporach for Investigating the Integration of E-Mobility Charging Infrastructure. 2nd International symposium on Environment Friendly Energies and Applications- EFEA, Newcastle upon Tyne, UK, pp. 7487.

ElBanhawy, E. Y., R. Dalton, E.M.Thompson, 2012 B. RealTime E-Mobility Simulation in Metropolitan Area, 30 th International eCAADe Conference, Prague, volume 1, pp. 533-544

ElBanhawy, E. Y., R. Dalton, K. Nassar, 2013. A Integrating Space-Syntax And Discrete-Event Simulation For E-Mobility Analysis, AEI Conference, 2013, Penn State, Pennsylvaina, USA, volume 1, pp. 934-945

ELEMENT ENERGY LTD 2009. Strategies for the uptake of electric vehicles and associated infrastructure implications. In: CHANGE, T. C. O. C. (ed.). Cambridge, UK

HM Government, The Carbon Plan: Delivering Our Low Carbon future, Department of Energy and Climate Change: London, 2011.

Jacob Schluman,March 2011 "Israel gets its first EV battery swap station, makes charging stations obsolete", Israel, http://www.engadget.com/2011/03/31/israel-gets-its-first-evbattery-swap-station-makes-charging-st/(April 2013).

JATO, Incentives Fail to Simulate European Electric Vehicles Sales, 2011.

J. Gould and T. Golob, "Consumer E-Commerce, Virtual Accessibility and Sustainable Transport", Paper presented at the Social Change and Sustainable Transport, University of California, Berkeley, 28th March 2000.

M. McGuinness, May 2011, Majority of Consumers Ready to Consider Buying Plug-in Electric Vehicles, But Challenge Utilities with their Car Charging Demands, Accenture Study Finds", Accenture, 2011 ,https://newsroom.accenture.com/article_display.cfm?article_id $=520$ (April 2013).

M. Eppstein, Grover, K. David, Marshall, S. Jeffrey, Rizzo, M. Donna, "An agent-based model to study market penetration of plug-in hybrid electric vehicles". Ener. Pol., Vol. 39(6), pp. 3789-3802, 2011.

M. Nilsson, "Electric Vehicles: The phenomenon of range anxiety", Elvire, Vol. 1, pp. 16, Gothenburg: Lindholmen Science Park, 2011.

S. Jabbar, S. Edelkamp, and T. Willhalm, IGeometric travel planning," IEEE Transactions on Intelligent Transportation Systems, vol. 6, pp. 5\{16, March 2005.

S. McDonald, Practical issues with implementing smart charging: experiences from NAREC and Charge your Car. Presentation at the NSR e-Mobility project (Interreg IVB North Sea Region Programme), 30 March 2012 - Transnational EMobility NSR Expert Seminar and Public Event at Northumbria 
International Archives of the Photogrammetry, Remote Sensing and Spatial Information Sciences,

Volume XL-4/W1, 29th Urban Data Management Symposium, 29 - 31 May, 2013, London, United Kingdom

University, Newcastle upon Tyne, 2012. http://e-mobilitynsr.eu/event/e-mobility-nsr-partner-meeting-and-transnationalmeeting/

T. Hegazy, 1. M. (1999). Evosite: Evolution-based model for site layout planning. Journal of computing in civil engineering, July.

T. Preuss and J.-H. Syrbe, \An integrated tra \pm c information system," in Proc. 6th Int. Conf. Appl. Computer Networking in Architecture, Construction, Design, Civil Eng., and Urban Planning (europIA '97), 1997.

T. Willhalm, Engineering Shortest Paths and Layout Algorithms for Large Graphs, Doctoral Dessertation, Feb 2005.

U. Brandes, F. Schulz, D. Wagner, and T. Willhalm, IGenerating node coordinates for shortest-path computations in transportation networks," ACM Journal on Experimental Algorithmics, vol. 9, no. 1, p. R1, 2004. 